\title{
Internal quantum efficiency of III-nitride quantum dot superlattices grown by plasma-assisted molecular-beam epitaxy
}

\author{
Ž. Gačević, ${ }^{1,2}$ A. Das, ${ }^{1}$ J. Teubert, ${ }^{1,3}$ Y. Kotsar, ${ }^{1}$ P. K. Kandaswamy, ${ }^{1}$ Th. Kehagias, ${ }^{4}$ \\ T. Koukoula, ${ }^{4}$ Ph. Komninou, ${ }^{4}$ and E. Monroy ${ }^{1, a)}$ \\ ${ }^{1}$ CEA-CNRS Group "Nanophysique et Semiconducteurs," CEA-Grenoble, INAC/SP2M 17 rue des Martyrs, \\ 38054 Grenoble, France \\ ${ }^{2}$ ISOM and Dpt. de Ingeniería Electrónica, ETSI Telecomunicación, Universidad Politécnica de Madrid, \\ Avda. Complutense s/n, 28040 Madrid, Spain \\ ${ }^{3}$ I. Physikalisches Institut, Justus-Liebig-Universitaet Giessen, 35392 Giessen, Germany \\ ${ }^{4}$ Physics Department, Aristotle University of Thessaloniki, GR 54124 Thessaloniki, Greece
}

\begin{abstract}
We present a study of the optical properties of GaN/AlN and InGaN/GaN quantum dot (QD) superlattices grown via plasma-assisted molecular-beam epitaxy, as compared to their quantum well $(\mathrm{QW})$ counterparts. The three-dimensional/two-dimensional nature of the structures has been verified using atomic force microscopy and transmission electron microscopy. The QD superlattices present higher internal quantum efficiency as compared to the respective QWs as a result of the three-dimensional carrier localization in the islands. In the QW samples, photoluminescence $(\mathrm{PL})$ measurements point out a certain degree of carrier localization due to structural defects or thickness fluctuations, which is more pronounced in $\mathrm{InGaN} / \mathrm{GaN}$ QWs due to alloy inhomogeneity. In the case of the QD stacks, carrier localization on potential fluctuations with a spatial extension smaller than the QD size is observed only for the InGaN QD-sample with the highest In content (peak emission around $2.76 \mathrm{eV}$ ). These results confirm the efficiency of the QD three-dimensional confinement in circumventing the potential fluctuations related to structural defects or alloy inhomogeneity. PL excitation measurements demonstrate efficient carrier transfer from the wetting layer to the QDs in the GaN/AlN system, even for low QD densities $\left(\sim 10^{10}\right.$ $\mathrm{cm}^{-3}$ ). In the case of InGaN/GaN QDs, transport losses in the GaN barriers cannot be discarded, but an upper limit to these losses of $15 \%$ is deduced from PL measurements as a function of the excitation wavelength.
\end{abstract}

\section{INTRODUCTION}

InGaN/GaN heterostructures have been a subject of extensive interest due to their application in blue/green/white light-emitting diodes and laser diodes. Their electroluminescence properties, surprisingly insensitive to the presence of a high dislocation density $\left(\sim 10^{9} \mathrm{~cm}^{-2}\right)$, have led to strong controversy about the light emission mechanisms and their correlation with structural properties. ${ }^{1-5}$ To assess the optical quality of these structures, the internal quantum efficiency (IQE) is often used as a reference parameter. IQE is defined as IQE $=R_{\mathrm{R}} /\left(R_{\mathrm{R}}+R_{\mathrm{NR}}\right)$, with $R_{\mathrm{R}}$ and $R_{\mathrm{NR}}$ being the radiative and nonradiative recombination rates, respectively. IQE is often estimated as the ratio between the photoluminescence (PL) at room temperature (RT) and that at low temperatures $(T<10 \mathrm{~K})$. However, there is a huge dispersion in the reported values of IQE, and their interpretation is the object of intense discussion. High values of IQE are often attributed to phase separation in InGaN quantum wells ${ }^{6-8}$ (QWs): Due to the strong localization in In-rich clusters, carriers can no longer diffuse toward nonradiative recombination centers. The effect of such alloy fluctuations can be enhanced by the polarization-related internal electric field. ${ }^{9}$ The possible role of V-shaped pit defects in circumventing carrier dislocations

\footnotetext{
a) Author to whom correspondence should be addressed. Electronic mail: eva.monroy@cea.fr.
}

and preventing nonradiative recombination has also been discussed. ${ }^{10}$

In order to control and fully exploit the advantages of carrier localization, several groups reported the controlled fabrication of self-assembled InGaN quantum $\operatorname{dots}^{11-16}$ (QDs) and GaN QDs, ${ }^{17-23}$ making use of the lattice-mismatch-induced Stranski-Krastanov growth mode. Due to the presence of strong compressive strain, a film of a few monolayers (ML) of $\mathrm{GaN}(\mathrm{InGaN})$ on $\mathrm{AlN}(\mathrm{GaN})$ tends to relax elastically via the formation of three-dimensional (3D) islands interconnected by a thin ( $\sim 1$ to $2 \mathrm{ML}$ ), highly strained twodimensional (2D) wetting layer. Unlike with lithographic techniques, the elastic nature of Stranski-Krastanov relaxation leads to defect-free objects, small enough to provide 3D carrier confinement. As a result, the excitons trapped in QDs are expected to be much more insensitive to nonradiative recombination than are those in QW structures. ${ }^{11-13,24,25}$

The aim of this paper is to give better insight into how the structural differences between InGaN/GaN and GaN/AlN QW and QD superlattices (SLs) affect the luminescence stability, and consequently the IQE, of III-nitride quantum confined structures.

\section{SAMPLE GROWTH}

GaN/AlN QW and QD 40-period SLs with various GaN QW/QD dimensions were deposited on 1- $\mu \mathrm{m}$-thick 
(0001)-oriented AlN-on-sapphire templates via plasmaassisted molecular-beam epitaxy. The nitrogen-limited growth rate was fixed at $0.3 \mathrm{ML} / \mathrm{s}(\sim 270 \mathrm{~nm} / \mathrm{h})$, and the substrate temperature, measured by a thermocouple in mechanical contact with the molybdenum sample holder, was $T_{\mathrm{S}}=720^{\circ} \mathrm{C}$. GaN/AIN QW structures, with a barrier thickness of $7 \mathrm{~nm}$ and a QW thickness varying between $1.25 \mathrm{~nm}$ and $3 \mathrm{~nm}$, were grown under Ga-rich conditions without growth interruptions, as described in Refs. 26 and 27. Highresolution transmission electron microscopy (HRTEM) studies showed that the GaN/AlN interfaces were chemically abrupt at the atomic layer scale, and that thickness fluctuations were limited to \pm 1 atomic layer. ${ }^{28}$

The synthesis of polar GaN/AlN QDs was performed using one of two methods: GaN deposition under N-rich conditions $^{24}$ (samples emitting at wavelengths shorter than $360 \mathrm{~nm}$ ) or GaN deposition under Ga-rich conditions followed by a growth interruption ${ }^{20}$ (samples emitting at wavelengths longer than $360 \mathrm{~nm}$ ). N-rich growth implies a reduction of the mobility of the adsorbed species during growth that results in a high density $\left(10^{11}\right.$ to $\left.10^{12} \mathrm{~cm}^{-2}\right)$ of small QDs (1 to $2 \mathrm{~nm}$ high). In contrast, Ga-rich conditions enhance the adatom mobility, leading to a lower QD density $\left(10^{10}\right.$ to $\left.10^{11} \mathrm{~cm}^{-2}\right)$ and bigger QDs ( 2 to $5 \mathrm{~nm}$ high). The difference between these growth techniques is illustrated by the atomic force microscopy (AFM) images in Fig. 1, which present QDs resulting from the deposition of $4 \mathrm{ML}$ of GaN (a) under N-rich conditions and (b) under Ga-rich conditions. The samples under study present PL peak wavelengths varying from 310 to $450 \mathrm{~nm}$ corresponding to QD heights varying from 1 to $3 \mathrm{~nm}$.

We also fabricated a series of InGaN/GaN QW and QD 20-period SLs deposited on $4-\mu \mathrm{m}$-thick and $10-\mu \mathrm{m}$-thick GaN-on-sapphire templates, respectively. In the case of the QWs, the Ga flux was fixed at $30 \%$ of the stoichiometric value, and the In flux was tuned to have two monolayers of excess In at the growth front. ${ }^{29}$ For the growth of the $3 \mathrm{~nm}$ thick GaN barriers, a second Ga cell was used at the stoichometric temperature, and the In shutter was kept open in order to guarantee $2 \mathrm{D}$ growth. The structures were synthesized without growth interruptions. Several samples were grown at various substrate temperatures between $610^{\circ} \mathrm{C}$ and $640{ }^{\circ} \mathrm{C}$. The morphology and structural properties of these samples were investigated via scanning transmission electron microscopy (STEM), as illustrated in Fig. 2. The 20 periods of the

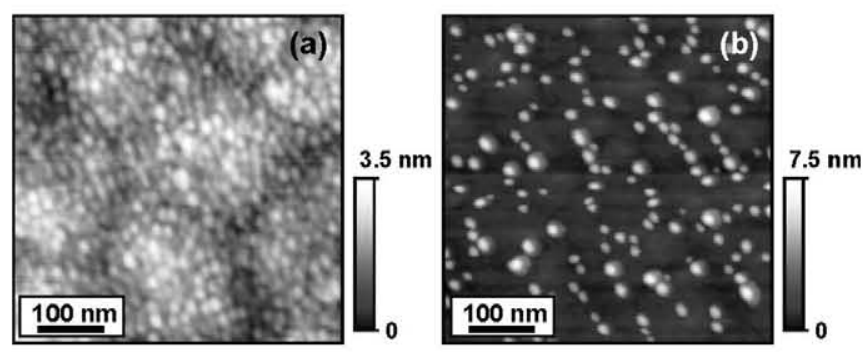

FIG. 1. (Color online) AFM images of GaN/AIN QDs synthesized by the deposition of $4 \mathrm{ML}$ of GaN under (a) N-rich and (b) Ga-rich conditions. Note that N-rich conditions lead to a higher density of smaller QDs, whereas Ga-rich conditions lead to a lower density of bigger QDs.

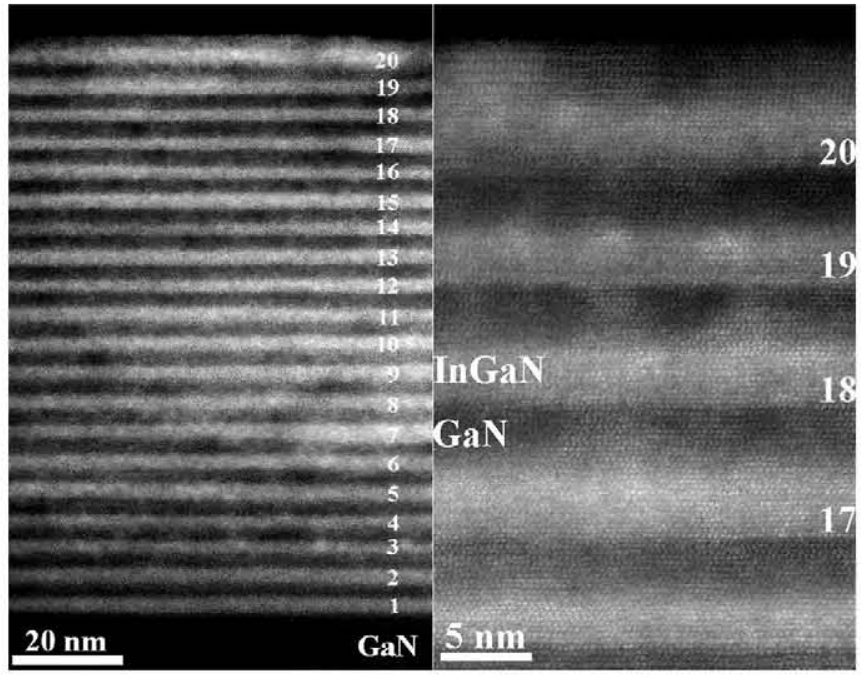

FIG. 2. Cross-sectional STEM images of InGaN/GaN QWs. Left: Overall view of the 20-period superlattice, in which the InGaN QWs present with brighter contrast because they comprise the heavier element. Right: A magnified part of the image on the left showing the atomically flat $\mathrm{GaN} / \mathrm{InGaN}$ interfaces and the interdiffusion at the $\mathrm{InGaN} / \mathrm{GaN}$ interfaces along the growth axis.

InGaN/GaN QWs are clearly visible (Fig. 2, left). In the magnified image on the right, atomically sharp $\mathrm{GaN} / \mathrm{InGaN}$ interfaces along the growth axis are observed, whereas the InGaN/GaN interfaces present interdiffusion with an average value of $0.7 \pm 0.1 \mathrm{~nm}$. Let us remind the reader that a certain degree of interdiffusion is commonly observed in this kind of structure grown by molecular-beam epitaxy..$^{30,31}$

For the generation of InGaN QDs, the Ga flux was fixed at $30 \%$ of the stoichiometric value, and the In flux was tuned close to the stoichiometry. Therefore, the Stranski-Krastanov transition was forced by the lattice mismatch, in spite of the slightly metal-rich atmosphere and the well-known surfactant effect of In, which promotes $2 \mathrm{D}$ growth. ${ }^{32}$ For the growth of the $6 \mathrm{~nm}$ spacer, the In shutter was closed and the Ga flux was fixed at the stoichiometric value. Figures 3(a) and 3(b) compare AFM images of InGaN/GaN QDs and the 2D surface of InGaN/GaN QWs, respectively. In HRTEM observations taken along the $[11 \overline{2} 0]_{\mathrm{GaN}}$ zone axis, a truncated pyramidal-shaped surface and embedded QDs of wurtzite structure are identified (Fig. 4). Although the shape of the surface QDs is well defined, the embedded QDs are less clear due to thickness effects from projected material overlap and scattered contrast from beam-induced partial indium clustering. In general, the projected diameter of the QDs ranges

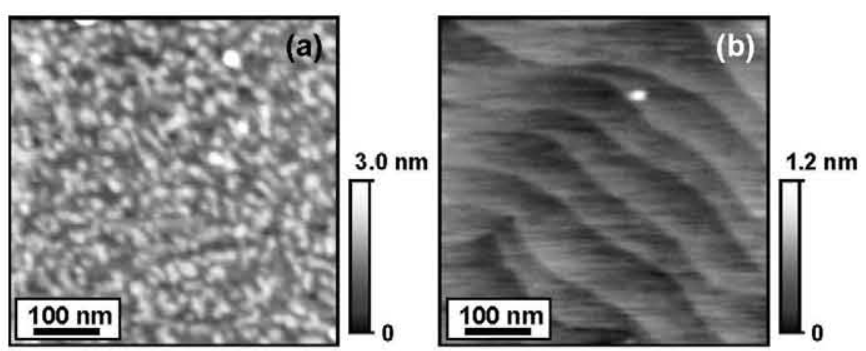

FIG. 3. (Color online) AFM images of (a) $\mathrm{InGaN} / \mathrm{GaN}$ QDs as compared to (b) $\mathrm{InGaN} / \mathrm{GaN} \mathrm{QWs}$. 


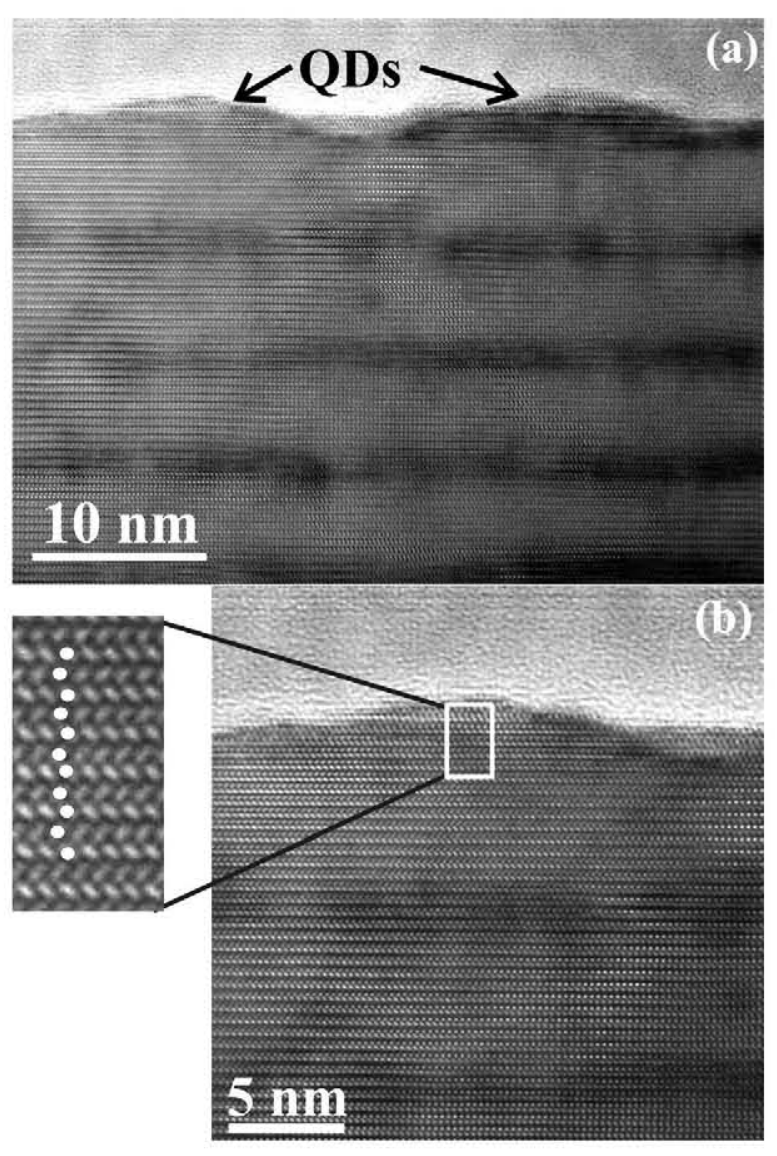

FIG. 4. HRTEM images of InGaN QDs viewed along the [1120] zone axis. (a) Surface and embedded QDs are detected along with the wetting layers. (b) A magnified view of a surface QD depicting its wurtzite structure in atomic scale.

from 17 to $25 \mathrm{~nm}$, and their height is measured as $\sim 2.2 \pm 0.2 \mathrm{~nm}$. The wetting layers are also visible.

\section{OPTICAL CHARACTERIZATION}

For PL experiments, the samples were mounted in a cold-finger cryostat with the temperature controlled from $T=7 \mathrm{~K}$ to RT. PL was excited with a frequency-doubled argon laser $(\lambda=244 \mathrm{~nm})$ and collected into a JobinYvon HR460 monochromator equipped with an ultravioletenhanced charge-coupled device (CCD) camera. The diameter of the excitation spot on the sample was about $100 \mu \mathrm{m}$. The excitation power was kept around $100 \mu \mathrm{W}$, low enough to avoid screening of the internal electric field. The lowtemperature ( $T=7 \mathrm{~K}$ ) PL spectra of the samples under study are presented in Fig. 5. In the case of the GaN/AIN QWs, the spectral structure of the emission is due to monolayer thickness fluctuations in the QWs, as described elsewhere. ${ }^{33}$ In the rest of the structures (GaN/AlN QDs and InGaN/GaN QDs and QWs), the broader linewidth makes it possible to observe the superimposition of a Fabry-Perot interference pattern related to the total nitride thickness.

The evolution of the integrated PL intensity as a function of temperature, normalized by the integrated PL intensity at low temperature $(T=7 \mathrm{~K}$ ), is presented in Fig. 6 for $\mathrm{GaN} / \mathrm{AIN}$ and $\mathrm{InGaN} / \mathrm{GaN}$ QWs and QDs. Keeping in mind that the emission intensity remains stable below $25 \mathrm{~K}$ for all
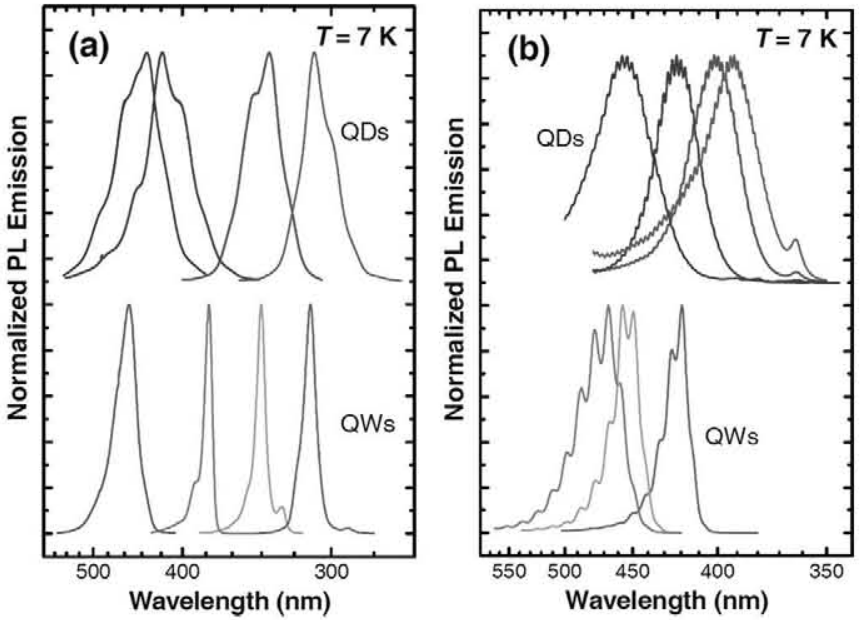

FIG. 5. (Color online) The normalized photoluminescence spectra of (a) GaN/AIN and (b) InGaN/GaN QD and QW superlattices. The spectra are vertically shifted for clarity.

of the samples, the values presented in Fig. 6 should correspond directly to the IQE at different temperatures. These results confirm the improved thermal stability of QDs over QWs, as a result of the $3 \mathrm{D}$ carrier confinement, in agreement with previous reports. ${ }^{11-13,24}$ Moreover, the thermal stability of the GaN/AIN QD nanostructures is significantly better than that of the InGaN/GaN QD nanostructures, which is explained by the stronger 3D localization stemming from the larger band offsets.

The thermal evolution of the PL is characterized not only by the intensity decline due to the activation of nonradiative recombination processes, but also by a spectral shift, which can provide information about the carrier localization in potential fluctuations. Figure 7 presents the evolution of the PL peak energy as a function of temperature in the cases of (a) GaN/AlN QWs, (b) GaN/AlN QDs, (c) InGaN/GaN QWs, and (d) InGaN/GaN QDs. The evolution of the PL peak energy from the GaN/AIN QDs fits well the evolution of the GaN bandgap calculated using Varshni's equation: $E_{\mathrm{G}}(T)=E_{\mathrm{G}}(T=0 \mathrm{~K})-\alpha T^{2} /(T+\beta)$, with $\alpha=0.59 \mathrm{meV} / \mathrm{K}$
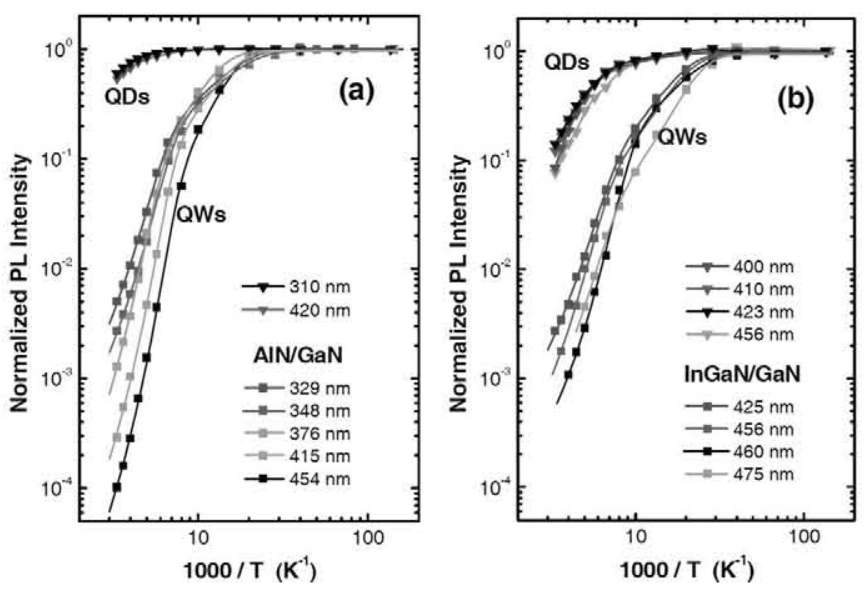

FIG. 6. (Color online) The temperature evolution of the integrated PL emission of (a) GaN/AIN and (b) InGaN/GaN QW and QD samples emitting at different wavelengths. Solid lines are fits to Eq. (5), neglecting transport losses, i.e., $T E(T)=1$. 

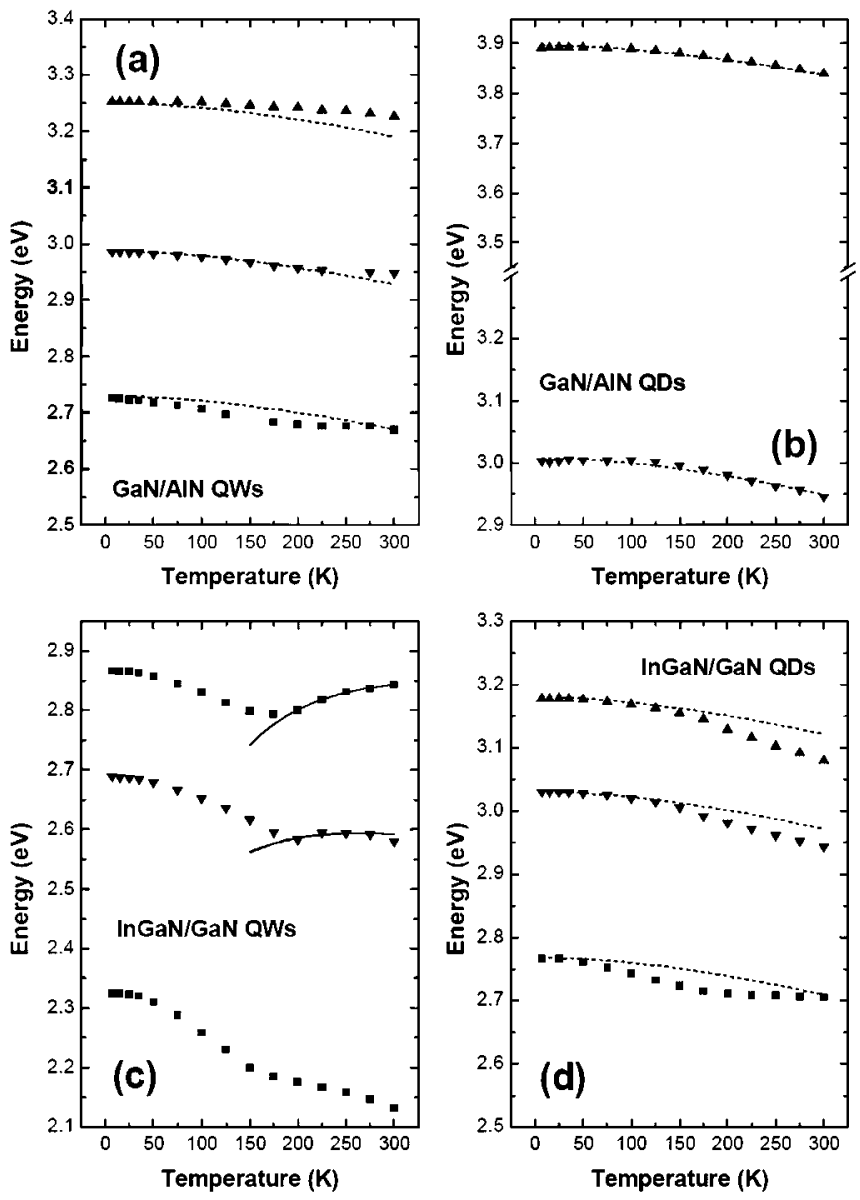

FIG. 7. (Color online) The temperature dependence of the PL peak position for (a) GaN/AlN QWs, (b) GaN/AlN QDs, (c) InGaN/GaN QWs, and (d) InGaNGaN QDs. Note that all of the figures have the same vertical span. The dashed lines in parts (a), (b), and (d) represent the evolution of the emission with temperature following Varshni's equation. The solid lines in (c) are fits to Eliseev's correction (Ref. 36) to model InGaN alloy fluctuations.

and $\beta=600 \mathrm{~K}$ for GaN (Ref. 37) (dashed curves in Fig. 7), which indicates that potential fluctuations inside the QDs are negligible. This is in contrast with the intra-dot localization reported in the case of nonpolar QDs, ${ }^{38}$ which can be attributed to the presence of structural defects. Also in polar InGaN/GaN QDs emitting in the 3.0-2.85 eV spectral range, Lefebvre et al. reported carrier localization by potential fluctuations with a spatial extension much smaller than the QD size. ${ }^{34}$ In our case, a deviation from Varshni's equation associated to $\mathrm{InGaN} / \mathrm{GaN}$ intra-dot localization is resolved only in the QD sample emitting at $2.76 \mathrm{eV}$ at low temperature, which points to a more uniform In distribution in the QDs with a lower In content (3.2-3.0 eV spectral range).

In the QW samples, the evolution of the emission peak energy as a function of temperature describes an $\mathbf{S}$ shape, which is particularly marked for the InGaN/GaN system. This S-shaped variation is associated with potential fluctuations in the QWs: the blueshift at intermediate temperatures is explained by the filling of potential valleys with different depths upon excitation. ${ }^{2,54-36}$ In the case of GaN/AlN QWs, the potential fluctuations responsible for this behavior can be related to variations in the QW thickness or/and to the presence of structural defects. The remarkable enhancement of the S-shape in InGaN QWs points to alloy inhomogeneities. For quantification of the potential fluctuations, Eliseev et al. ${ }^{36}$ proposed a band-tail model assuming a Gaussianlike distribution of the density of states, which results in a correction to Varshni's equation by $-\sigma^{2} / k T$, where $\sigma$ is dispersion of the Gaussian band-tail density of states. From the analysis of InGaN/GaN QWs, values of $\sigma=40 \pm 15 \mathrm{meV}$ are obtained, which are comparable to typical measurements in InGaN light emitting diodes. ${ }^{36}$

These results confirm the efficiency of the 3D confinement provided by the QDs in screening the potential fluctuations related to dislocations, V-defects, or alloy fluctuations existing in the QW structures.

\section{DISCUSSION}

In order to correctly interpret the thermal evolution of the PL intensity (Fig. 6), let us remind the reader that PL is a consequence of three successive processes: electron-hole generation (photon absorption), thermalization (phonon emission), and radiative recombination (photon emission). The generation and thermalization processes can be considered as insensitive to temperature within the scope of our analysis. The external quantum efficiency can be decomposed into three contributing terms: transport efficiency $(T E)$, recombination efficiency $(R E)$, and extraction efficiency. In general, both transport and recombination efficiency are sensitive to temperature variation, whereas the thermal sensitivity of extraction efficiency can be neglected, because it depends mainly on the geometry of the structure, which is barely influenced by temperature.

The recombination efficiency can be written as

$$
R E=\frac{R_{\mathrm{R}}}{R_{\mathrm{R}}+R_{\mathrm{NR}}}=\frac{1}{1+\left(R_{\mathrm{NR}} / R_{\mathrm{R}}\right)},
$$

where $R_{\mathrm{R}}$ and $R_{\mathrm{NR}}$ denote the radiative and nonradiative recombination rates, respectively. Making use of simple rate equations ${ }^{39}$ under (optical) injection,

$$
\frac{\partial n}{\partial t}=G-\frac{n}{\tau_{\mathrm{R}}}-\frac{n}{\tau_{\mathrm{NR}}}
$$

where $n$ is the minority carrier or exciton density concerned, $G$ is the generation rate, and $\tau_{\mathrm{R}}$ and $\tau_{\mathrm{NR}}$ are the radiative and nonradiative lifetimes, respectively. Considering that nonradiative recombination centers are thermally activated, i.e., $\tau_{\mathrm{NR}}=\tau_{0} e^{E_{a} / k T}$, and that the steady-state population of minority carriers governs the PL intensity (i.e., $I_{\mathrm{PL}} \propto n / \tau_{\mathrm{R}}$ ), we arrive at the expression

$$
R E=\frac{1}{1+a e^{-E_{a} / k T}},
$$

where

$$
a=\tau_{R} / \tau_{0} .
$$

The presence of carrier localization in potential fluctuations, as observed in GaN/AlN and $\mathrm{InGaN} / \mathrm{GaN}$ QWs, complicates 
rate equation (2) by introducing an additional thermal dependence, ${ }^{1}$

$$
R E=\frac{1}{\left(1+a_{1} e^{-E_{a} / k T}\right)\left(1+a_{2} e^{\left.-E_{\mathrm{loc} / k T}\right)}\right.},
$$

with $E_{\text {loc }}$ being the average localization energy.

Taking the previous approximations into account, the PL intensity ratio between high temperature and low temperature can be expressed as

$$
\frac{I_{\mathrm{PL}}(T)}{I_{\mathrm{PL}}(T=0)} \approx \frac{T E(T)}{\left(1+a_{1} e^{-E_{a} / k T}\right)\left(1+a_{2} e^{-E_{\mathrm{loc}} / k T}\right)} .
$$

Therefore, the temperature dependent PL experiment cannot distinguish between transport and nonradiative recombination losses.

A variation in the excitation energy can affect the generation process and the transport efficiency while the recombination efficiency remains unaffected, because photoexcited carriers rapidly thermalize to the lowest energy state, where they usually remain for a period of time orders of magnitude longer than the thermalization time. This implies that the carriers lose information about the initial excitation state before they recombine. Figure 8 schematically depicts carrier transport under optical injection when the barriers are excited. Note that the carriers recombining radiatively in the QDs originate from the barriers, from the wetting layer, or from the QDs themselves. Transport losses can be significant for the carriers generated in the barriers or in the wetting layers.

To gain some insight into the carrier absorption and transfer mechanisms, we performed photoluminescence excitation (PLE) spectroscopy measurements on GaN/AlN and InGaN/GaN QDs. PLE was carried out using a tunable excitation source consisting of a $500 \mathrm{~W}$ Xe lamp coupled to a Gemini 180 monochromator; the PL was analyzed using a Triax 550 monochromator and was detected by either a CCD camera or a photomultiplier tube operating in the photon counting mode. The excitation power density was about 200 $\mu \mathrm{W} / \mathrm{cm}^{2}$ at $350 \mathrm{~nm}$. The PLE measurements have been linearly corrected to account for the spectral variations of the

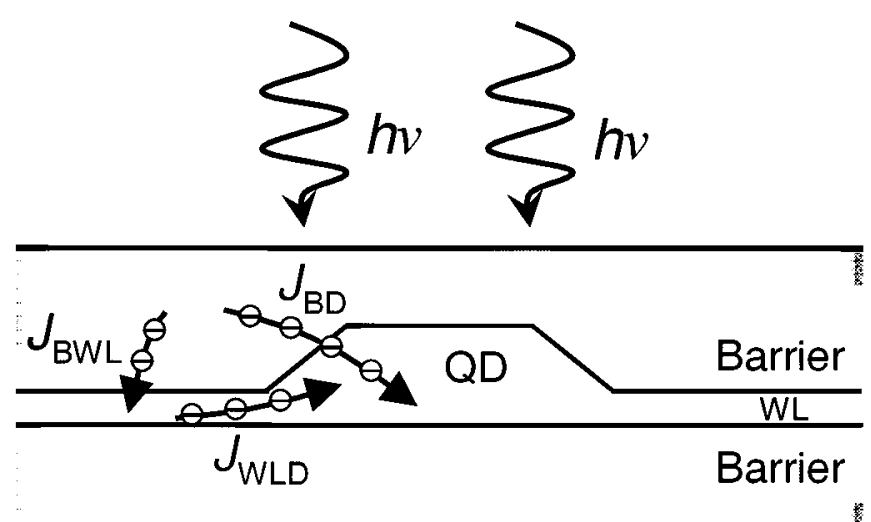

FIG. 8. (Color online) Carrier diffusion paths in QD structures under optical injection: $J_{\mathrm{BWL}}, J_{\mathrm{BD}}$, and $J_{\mathrm{WLD}}$ denote the carrier transport between barrier and wetting layer, between the barrier and the $\mathrm{QD}$, and between the wetting layer and the QD, respectively.

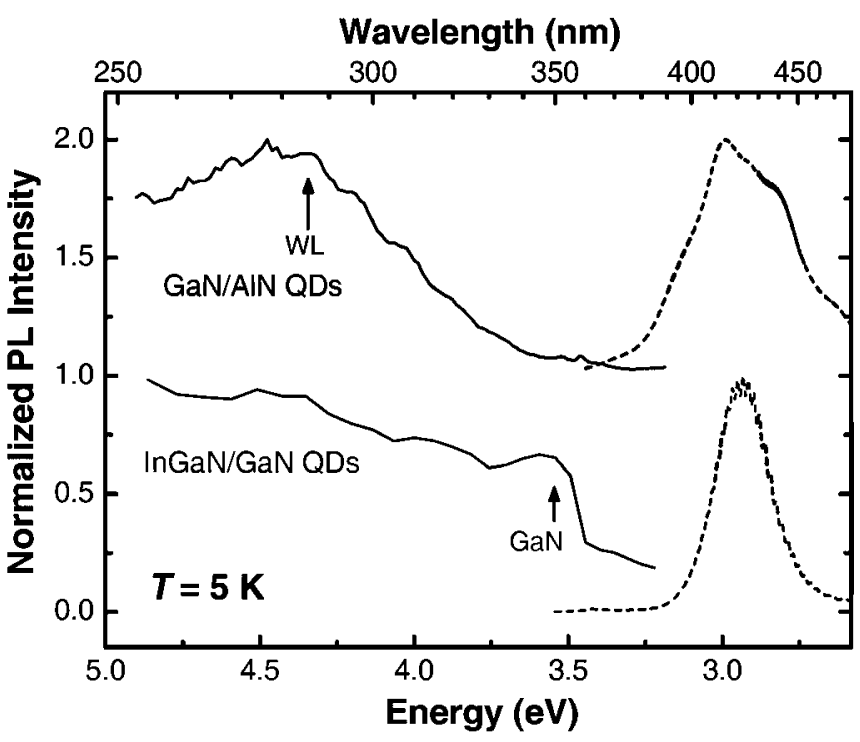

FIG. 9. PLE (solid lines) and PL (dashed lines) spectra from GaN/AlN and InGaN/GaN QDs. The signature of the wetting layer in low density GaN/ AlN QDs and the absorption in GaN barriers in InGaN/GaN QDs are indicated by arrows.

excitation intensity and corrected for the spectral response of the system. Figure 9 displays the PL and PLE spectra measured for GaN/AlN and InGaN/GaN QDs at $T=5 \mathrm{~K}$. In the case of the GaN/AIN QDs, we observe a strong enhancement of the PL intensity when exciting above the wetting layer level $(\sim 4.1 \mathrm{eV})$, in agreement with previous reports, ${ }^{23}$ whereas in the case of the InGaN/GaN QDs we observe an increase of the luminescence when exciting above the $\mathrm{GaN}$ barriers $(\sim 350 \mathrm{~nm})$.

The effect of the excitation wavelength on the IQE is illustrated in Fig. 10 for both GaN/AlN QDs and InGaN/ GaN QDs. The IQE is calculated as the ratio between the integrated PL intensity at RT and that at low temperature

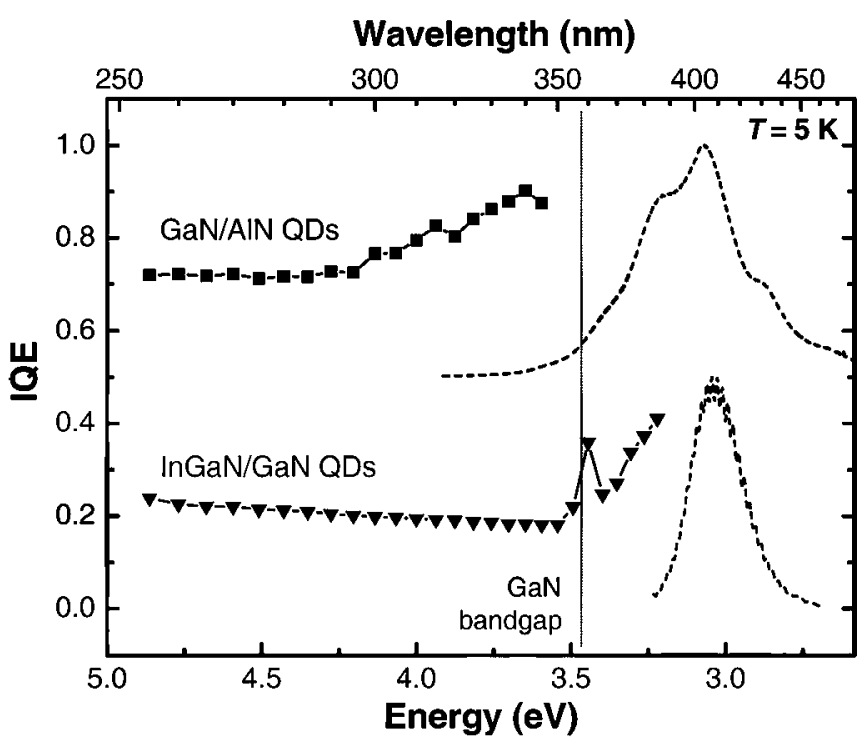

FIG. 10. IQE from GaN/AlN and InGaN/GaN QDs calculated as the ratio of the integrated PL intensity measured at $T=300 \mathrm{~K}$ and at $T=5 \mathrm{~K}$, as a function of the excitation wavelength. Normalized and vertically shifted lowtemperature $(T=5 \mathrm{~K}) \mathrm{PL}$ spectra are represented with dashed lines for reference. 
TABLE I. Values of $\mathrm{IQE}=I_{\mathrm{PL}}(\mathrm{RT}) / I_{\mathrm{PL}}(T=4 \mathrm{~K})$, activation energy of nonradiative processes $E_{a}$, and localization energy $E_{\mathrm{loc}}$ extracted from the analysis of the PL emission of InGaN/GaN and GaN/AIN QWs and QDs.

\begin{tabular}{lcccc}
\hline \hline Structure & InGaN/GaN QWs & GaN/AlN QWs & InGaN/GaN QDs & GaN/AlN QDs \\
\hline Number of samples & 6 & 5 & 8 & 3 \\
Emission range $(\mathrm{nm})$ & $430-530$ & $325-460$ & $390-410$ & $310-420$ \\
$E_{a}(\mathrm{meV})$ & $11 \pm 4$ & $15 \pm 3$ & $50 \pm 2$ & $80 \pm 5$ \\
$E_{\text {loc }}(\mathrm{meV})$ & 30 to $80^{\mathrm{a}}$ & $110 \pm 15$ & $\ldots$ & $\ldots$ \\
$\mathrm{IQE}$ & 0.0004 to 0.01 & 0.0001 to $0.02^{\mathrm{b}}$ & 0.1 to 0.6 & 0.3 to 0.8 \\
\hline \hline
\end{tabular}

${ }^{\mathrm{a}}$ Increasing for increasing PL peak wavelength.

${ }^{\mathrm{b}}$ Decreasing for increasing PL peak wavelength.

( $T=5 \mathrm{~K}$ ) as a function of the excitation wavelength using the Xe lamp setup. In the case of GaN QDs, the AlN barriers are never excited in our experimental conditions. The PLE experiments in Fig. 9 point out a significant contribution of carriers photo-generated in the wetting layer to the QD luminescence. However, the study of the $I_{\mathrm{PL}}(T=300 \mathrm{~K}) / I_{\mathrm{PL}}(0)$ ratio as a function of the excitation wavelength in Fig. 10 shows no variation in the excitation above and below the wetting layer level. We can therefore conclude that the transport losses from the wetting layer to the QDs are negligible.

The IQE of the InGaN/GaN QD samples is lower than the IQE of their GaN/AIN counterparts. We observe a slight increase of the IQE of InGaN QDs once the excitation energy is tuned below the GaN bandgap $(3.47 \mathrm{eV} \approx 357 \mathrm{~nm}$, at low temperature), which might be an indication of transport losses in the GaN barriers. Our results are consistent with the values reported by Sénès et al., ${ }^{13,40}$ who measured an IQE of $30 \%$ in InGaN/GaN QDs when exciting at the 325 $\mathrm{nm}$ line ( $\mathrm{GaN}$ barriers being excited) and $60 \%$ when exciting at the $390 \mathrm{~nm}$ line (GaN barriers not excited). These differences as a function of the excitation wavelength might be partially responsible for the high dispersion in reported IQE values of InGaN/GaN as compared to the case of GaN/AlN QDs. However, one must be cautious when assigning them to transport losses, because an enhancement of the PL intensity is observed when approaching resonant excitation, as is the case for GaN/AIN QDs in the $300-350 \mathrm{~nm}$ spectral range in Fig. 10. Therefore, regarding the InGaN/GaN QDs, we can state only that an upper limit of transport losses in the barriers can be estimated from $[\operatorname{IQE}(\lambda=370 \mathrm{~nm}) /$ $\operatorname{IQE}(\lambda=340 \mathrm{~nm})]-1 \approx 15 \%$.

Experimental measurements of the PL intensity as a function of temperature (Fig. 6) can be well fitted to Eq. (5), neglecting transport losses $(T E(T)=1)$. The obtained activation energies $\left(E_{a}\right)$ and localization energies $\left(E_{\mathrm{loc}}\right)$ are listed in Table I. It is important to note that the value of $E_{a}$ does not correspond to the band offset, although it is influenced by it. $E_{a}$ represents the energetic barrier that the carriers must surmount in order to reach the nonradiative recombination centers. In the case of QDs, the confinement in the dot increases this potential barrier. Higher temperatures are therefore required for carriers to escape, probably via the wetting layer. On the other hand, QD samples are well fitted with a single exponential, i.e., assuming negligible intra-dot localization effects, as expected from the results in Figs. 7(b) and 7(d). In contrast, QW samples require two activation energies in order to get a good fit of the thermal evolution of the PL intensity, which is consistent with the observed Sshaped evolution of the PL peak energy in Figs. 7(a) and $7(\mathrm{c})$.

\section{CONCLUSIONS}

In summary, making use of the Stranski-Krastanov growth mechanism, we successfully fabricated SLs of InGaN/GaN and GaN/AlN QDs using plasma-assisted molecular-beam epitaxy. The three-dimensional nature of the structures has been verified using atomic force microscopy and transmission electron microscopy. PL measurements confirm the superior internal quantum efficiency of the QD structures as compared to the respective QWs, as a result of the $3 \mathrm{D}$ carrier localization. In the QW samples, the S-shape described by the PL peak energy as a function of temperature indicates carrier localization in GaN/AlN QWs, attributed to structural defects or thickness fluctuations, and more markedly in InGaN/GaN, due to alloy inhomogeneity. In the case of the QD stacks, carrier localization in local potential minima with a spatial extension smaller than the QD size is observed only for the InGaN QDs with the highest In content (peak emission around $2.76 \mathrm{eV}$ ) under study. These results confirm the efficiency of the QD three-dimensional confinement in avoiding the potential fluctuations related to structural defects or alloy inhomogeneity. The PL excitation measurements demonstrate efficient carrier transfer from the wetting layer to the QDs in the GaN/AlN system, even for a low density of QDs $\left(\sim 10^{10} \mathrm{~cm}^{-3}\right)$. In the case of InGaN/GaN QDs, transport losses in the GaN barriers cannot be discarded, but an upper limit to these losses of $15 \%$ is deduced from PL measurements as a function of the excitation wavelength.

\section{ACKNOWLEDGMENTS}

Z.G. wants to thank his Ph.D. advisor Enrique Calleja. This work was partially supported by the European Commission within the 7th European Framework Projects "DOTSENSE" (Grant No. 224212) and "UNITRIDE" (Grant No. 233950). Z.G. acknowledges financial support from CAM (P2009/ESP-1503) and MICINN (MAT2008-04815).

${ }^{1}$ M. S. Minsky, S. Watanabe, and N. Yamada, J. Appl. Phys. 91, 5176 (2002).

${ }^{2}$ A. Sasaki, K. Nishizuka, T. Wang, S. Sakai, A. Kaneta, Y. Kawakami, and Sg. Fujita, Solid State Commun. 129, 31 (2004).

${ }^{3}$ C. E. Martinez, N. M. Stanton, A. J. Kent, D. M. Graham, P. Dawson, M. J. Kappers, and C. J. Humphreys, J. Appl. Phys. 98, 053509 (2005). 
${ }^{4}$ L. T. Tan, R. W. Martin, K. P. O’Donnell, and I. M. Watson, Appl. Phys. Lett. 89, 101910 (2006).

${ }^{5}$ C. Netzel, H. Bremers, L. Hoffmann, D. Fuhrmann, U. Rossow, and A. Hangleiter, Phys. Rev. B 76, 155322 (2007).

${ }^{6}$ S. Chichibu, T. Azuhata, T. Sota, and S. Nakamura, Appl. Phys. Lett. 69, 4188 (1996).

${ }^{7}$ Y. Narukawa, Y. Kawakami, M. Funato, S. Fujita, S. Fujita, and S. Nakamura, Appl. Phys. Lett. 70, 981 (1997).

${ }^{8}$ K. P. O'Donnell, R. W. Martin, and P. G. Middleton, Phys. Rev. Lett. 82, 237 (1999).

${ }^{9}$ M. Gallart, P. Lefebvre, A. Morel, T. Taliericio, B. Gil, J. Allègre, H. Mathieu, B. Damilano, N. Grandjean, and J. Massies, Phys. Status Solidi A $183,61(2001)$

${ }^{10}$ A. Hangleiter, F. Hitzel, C. Netzel, D. Fuhrmann, U. Rossow, G. Ade, and P. Hinze, Phys. Rev. Lett. 95, 127402 (2005)

${ }^{11}$ C. Adelmann, J. Simon, G. Feuillet, N. T. Pelekanos, B. Daudin, and G. Fishman, Appl. Phys. Lett. 76, 1570 (2000).

${ }^{12}$ B. Damilano, N. Grandjean, S. Dalmasso, and J. Massies, Appl. Phys. Lett. 75, 3751 (1999).

${ }^{13}$ M. Sénès, K. L. Smith, T. M. Smeeton, S. E. Hooper, and J. Heffernan, Phys. Rev. B 75, 045314 (2007).

${ }^{14}$ S. Gangopadhyay, Th. Schmidt, S. Einfeldt, T. Yamaguchi, D. Hommel, and J. Falta, J. Vac. Sci. Technol. B 25, 791 (2007).

${ }^{15}$ R. A. Oliver, M. J. Kappers, C. J. Humphreys, and G. A. D. Briggs, J. Appl. Phys. 97, 013707 (2005).

${ }^{16}$ K. Tachibana, T. Someya, and Y. Arakawa, Appl. Phys. Lett. 74, 383 (1999).

${ }^{17}$ B. Daudin, F. Widmann, G. Feuillet, Y. Samson, M. Arlery, and J. L. Rouvière, Phys. Rev. B 56, R7069 (1997).

${ }^{18}$ B. Damilano, N. Grandjean, F. Semond, J. Massies, and M. Leroux, Appl. Phys. Lett. 75, 962 (1999).

${ }^{19}$ S. Tanaka, M. Takeuchi, and Y. Aoyagi, Jpn. J. Appl. Phys., Part 2 39, L831 (2000).

${ }^{20}$ N. Gogneau, G. Jalabert, E. Monroy, E. Sarigiannidou, J.-L. Rouvière, T. Shibata, M. Tanaka, J.-M. Gérard, and B. Daudin, J. Appl. Phys. 96, 1104 (2004).

${ }^{21}$ J. Brown, F. Wu, P. M. Petroff, and J. S. Speck, Appl. Phys. Lett. 84, 690 (2004).

${ }^{22}$ A. Neogi, H. Everitt, H. Morkoç, T. Kuroda, and A. Tackeuchi, IEEE Trans. Nanotechnol. 4, 297 (2005)

${ }^{23}$ D. Simeonov, E. Feltin, J.-F. Carlin, R. Butté, M. Ilegems, and N. Grandjean, J. Appl. Phys. 99, 083509 (2006).
${ }^{24}$ F. Guillot, E. Bellet-Amalric, E. Monroy, M. Tchernycheva, L. Nevou, L. Doyennette, F. H. Julien, Le Si Dang, T. Remmele, M. Albrecht, T. Shibata, and M. Tanaka, J. Appl. Phys. 100, 044326 (2006)

${ }^{25}$ J. Renard, P. K. Kandaswamy, E. Monroy, and B. Gayral, Appl. Phys. Lett. 95, 131903 (2009).

${ }^{26}$ P. K. Kandaswamy, F. Guillot, E. Bellet-Amalric, E. Monroy, L. Nevou, M. Tchernycheva, A. Michon, F. H. Julien, E. Baumann, F. R. Giorgetta, D. Hofstetter, T. Remmele, M. Albrecht, S. Bilner, and Le Si Dang, J. Appl. Phys. 104, 093501 (2008)

${ }^{27}$ P. K. Kandaswamy, C. Bougerol, D. Jalabert, P. Ruterana, and E. Monroy, J. Appl. Phys. 106, 013526 (2009).

${ }^{28}$ E. Sarigiannidou, E. Monroy, N. Gogneau, G. Radtke, P. Bayle-Guillemaud, E. Bellet-Amalric, B. Daudin, and J. L. Rouvière, Semicond. Sci. Technol. 21, 912 (2006).

${ }^{29}$ E. Monroy, B. Daudin, E. Bellet-Amalric, N. Gogneau, D. Jalabert, F. Enjalbert, J. Brault, J. Barjon, and Le Si Dang, J. Appl. Phys. 93, 1550 (2003).

${ }^{30}$ P. Waltereit, O. Brandt, K. H. Ploog, M. A. Tagliente, and L. Tapfer, Phys. Rev. B 66, 165322 (2002).

${ }^{31}$ A. Dussaigne, B. Damilano, N. Grandjean, and J. Massies, J. Cryst. Growth 251, 471 (2003).

${ }^{32}$ J. Neugebauer, T. K. Zywietz, M. Scheffler, J. E. Northrup, H. Chen, and R. M. Feenstra, Phys. Rev. Lett. 90, 056101 (2003).

${ }^{33}$ M. Tchernycheva, L. Nevou, L. Doyennette, F. H. Julien, E. Warde, F. Guillot, E. Monroy, E. Bellet-Amalric, T. Remmele and M. Albrecht, Phys. Rev. B 73, 125347 (2006).

${ }^{34}$ P. Lefebvre, T. Taliercio, A. Morel, J. Allègre, M. Gallart, B. Gil, H. Mathieu, B. Damilano, N. Grandjean, and J. Massies, Appl. Phys. Lett. 78, 1538 (2001)

${ }^{35}$ Y.-H. Cho, G. H. Gainer, A. J. Fisher, J. J. Song, S. Keller, U. K. Mishra, and S. P. DenBaars, Appl. Phys. Lett. 73, 1370 (1997).

${ }^{36}$ P. G. Eliseev, P. Perlin, J. Lee, and M. Osinski, Appl. Phys. Lett. 71, 569 (1997).

${ }^{37}$ Y. Li, Y. Lu, H. Shen, M. Wraback, M. G. Brown, M. Schurman, L. Koszi, and R. A. Stall, Appl. Phys. Lett. 70, 2458 (1997).

${ }^{38}$ F. Rol, S. Founta, H. Mariette, B. Daudin, Le Si Dang, J. Bleuse, D. Peyrade, J.-M. Gérard, and B. Gayral, Phys. Rev. B 75, 125306 (2007).

${ }^{39}$ M. Leroux, N. Grandjean, B. Beaumont, G. Nataf, F. Semond, J. Massies, and P. Gibart, J. Appl. Phys. 86, 3721 (1999).

${ }^{40}$ M. Sénès, K. L. Smith, T. M. Smeeton, S. E. Hooper, and J. Heffernan, Physica E 40, 2066 (2008). 